\title{
Human Single-Chain Antibodies That Neutralize Elastolytic Activity of Pseudomonas aeruginosa LasB
}

\author{
Sirijan Santajit ${ }^{1,2}{ }^{\mathbb{D}}$, Thida Kong-ngoen ${ }^{1}$, Manas Chongsa-Nguan ${ }^{3}$, Usa Boonyuen ${ }^{4}$, Pornpan Pumirat ${ }^{1}$, \\ Nitat Sookrung ${ }^{5,6}$, Wanpen Chaicumpa ${ }^{5}$ and Nitaya Indrawattana ${ }^{1, *}$ \\ 1 Department of Microbiology and Immunology, Faculty of Tropical Medicine, Mahidol University, \\ Bangkok 10400, Thailand; sirijan.sa@wu.ac.th (S.S.); thida.kon@mahidol.ac.th (T.K.-n.); \\ pornpan.pum@mahidol.ac.th (P.P.) \\ 2 Department of Medical Technology, School of Allied Health Sciences, Walailak University, \\ Nakhon Si Thammarat 80160, Thailand \\ 3 Faculty of Public Health and Environment, Pathumthani University, Pathum Thani 12000, Thailand; \\ manas.cho@mahidol.ac.th \\ 4 Department of Molecular Tropical Medicine and Genetics, Faculty of Tropical Medicine, Mahidol University, \\ Bangkok 10400, Thailand; usa.boo@mahidol.ac.th \\ 5 Center of Research Excellence on Therapeutic Proteins and Antibody Engineering, Department of \\ Parasitology, Faculty of Medicine Siriraj Hospital, Mahidol University, Bangkok 10700, Thailand; \\ nitat.soo@mahidol.ac.th (N.S.); wanpen.cha@mahidol.ac.th (W.C.) \\ 6 Biomedical Research Incubator Unit, Department of Research, Faculty of Medicine Siriraj Hospital, \\ Mahidol University, Bangkok 10700, Thailand \\ check for \\ * Correspondence: nitaya.ind@mahidol.ac.th; Tel.: +66-2-354-9100 (ext. 1598)
}

updates

Citation: Santajit, S.; Kong-ngoen, T.; Chongsa-Nguan, M.; Boonyuen, U.;

Pumirat, P.; Sookrung, N.;

Chaicumpa, W.; Indrawattana, N.

Human Single-Chain Antibodies That

Neutralize Elastolytic Activity of

Pseudomonas aeruginosa LasB.

Pathogens 2021, 10, 765. https://

doi.org/10.3390/pathogens10060765

Academic Editors: Natalia Kirienko and Carolyn Cannon

Received: 24 May 2021

Accepted: 15 June 2021

Published: 17 June 2021

Publisher's Note: MDPI stays neutral with regard to jurisdictional claims in published maps and institutional affiliations.

Copyright: (c) 2021 by the authors. Licensee MDPI, Basel, Switzerland. This article is an open access article distributed under the terms and conditions of the Creative Commons Attribution (CC BY) license (https:// creativecommons.org/licenses/by/ $4.0 /)$.
Abstract: LasB (elastase/pseudolysin) is an injurious zinc-metalloprotease secreted by the infecting Pseudomonas aeruginosa. LasB is recognized as the bacterial key virulence factor for establishment of successful infection, acquisition of nutrients, dissemination, tissue invasion, and immune modulation and evasion. LasB digests a variety of the host tissue proteins, extracellular matrices, as well as components of both innate and adaptive immune systems, including immunoglobulins, complement proteins, and cytokines. Thus, this enzyme is an attractive target for disarming the P. aeruginosa. This study generated human single-chain antibodies ( $\mathrm{HuscFvs)}$ that can neutralize the elastolytic activity of native LasB by using phage display technology. Gene sequences coding HuscFvs (huscfus) isolated from HuscFv-displaying phage clones that bound to enzymatically active LasB were sub-cloned to expression plasmids for large scale production of the recombinant HuscFvs by the huscfv-plasmid transformed Escherichia coli. HuscFvs of two transformed E. coli clones, i.e., HuscFv-N42 and HuscFv$\mathrm{N} 45$, neutralized the LasB elastolytic activities in vitro. Computer simulation by homology modeling and molecular docking demonstrated that antibodies presumptively formed contact interfaces with the LasB residues critical for the catalytic activity. Although the LasB neutralizing mechanisms await elucidation by laboratory experiments, the HuscFvs should be tested further towards the clinical application as a novel adjunctive therapeutics to mitigate severity of the diseases caused by P. aeruginosa.

Keywords: elastase; elastolytic activity; human single-chain antibody; HuscFv; Pseudomonas aeruginosa; LasB; phage display technology

\section{Introduction}

LasB protease, known also as pseudolysin or elastase, is one of the toxic extracellular enzymes secreted by the infecting Pseudomonas aeruginosa via the bacterial type II secretion system [1,2]. This protease belongs to the M4 thermolysin family of zinc-dependent neutral metalloendopeptidases [3-8]. LasB causes destruction of the tissues and degradation of a variety of proteins of the infected mammalian hosts including elastin (elastolytic activity), casein, types III and IV collagens, fibronectin, etc.; all for establishment of successful 
bacterial infection, further invasion and dissemination, and nutrient acquisition [9-12]. Besides, the LasB promotes the P. aeruginosa infection by modulation and regulation of the host innate and adaptive immune responses. For example, LasB lyses fibronectin to expose the host receptors for the bacterial attachment; digests serum- $\alpha 1$-proteinase inhibitor, surfactant proteins $\mathrm{A}$ and $\mathrm{D}$, and bronchial mucosal proteinase inhibitors to disrupt the respiratory epithelium and destroys the ciliary function; digests human immunoglobulins (IgG and $\operatorname{Ig} \mathrm{A}$ ) and complement proteins; represses gamma interferon and tumor necrosis factor; disrupts alveolar macrophage activity by downregulation of reactive oxygen species generation to interrupt the bacterial killing [13-17]. The enzyme has also strong hemorrhagic activity and muscle destructive effects [18]. It involves in pathology of a variety of diseases caused by $P$. aeruginosa, including lung infections by increasing lung permeability, impairment of apoptotic cell clearance in cystic fibrosis and bronchiectasis, and cleavage of surfactant proteins $[17,19-21]$; chronic ulcer by degrading human skin proteins and wound fluids [22]; and corneal infection by causing corneal liquefaction, which can damage the vision functions [23].

Because the LasB protease plays key pathogenic roles during P. aeruginosa infection, the enzyme is one of the potential therapeutic targets for mitigation of the pseudomonal disease severity [24-26]. Previous data have shown that rabbits infected with a $\triangle$ lasB mutant strain displayed decreased severity of $P$. aeruginosa-mediated corneal ulceration [27]. Deletion of the las B gene resulted in less invasive P. aeruginosa infection in both mouse and Caenorhabditis elegans models, when compared to the infection caused by the wild-type strain $[28,29]$. Several antimicrobials and inhibitors have been used to block the synthesis of $P$. aeruginosa proteins (including LasB) that are crucial for bacterial survival and pathogenicity, i.e., kirromycin, pulvomycin, macrolides, clindamycin, chloramphenicol, aminoglycosides, tetracyclines, and synthetic oxazolidinone such as linezolid indole dipeptides, benzimidazole amidines, 2-arylbenzimidazoles, $\mathrm{N}$-substituted imidazoles, guanidines, and fusidic acid [30-32]. Moreover, several inhibitors have been developed as the LasB inhibitors including 10-phenanthroline-5,6-dione (phendione) and its derivatives, hydroxamate-based MMP inhibitors, and N-mercaptoacetyl-Phe-Tyr-amide [24,33,34]. Nevertheless, P. aeruginosa has multiple strategies to resist the antimicrobial drugs and becomes a member of "ESKAPE" (an acronym for the group of six highly virulent and antibiotic resistant Gram-positive and Gram-negative bacteria that include Enterococcus faecium, Staphylococcus aureus, Klebsiella pneumoniae, Acinetobacter baumannii, Pseudomonas aeruginosa, and Enterobacter species). The LasB chemical inhibitors tend to be toxic to mammalian cells, which limits their therapeutic usage. In this study, engineered human monoclonal singlechain antibodies [HuscFvs], which are small molecules [consist of only variable heavy chain domain $(\mathrm{VH})$ and variable light chain domain $(\mathrm{VL})$ linked together via a $\left(\mathrm{Gly}_{4} \mathrm{Ser}\right)_{3}$ peptide; VH-linker-VL] that bind to and neutralize elastolytic activity of the $P$. aruginosa LasB were generated using phage display technology and a human single-chain antibody (HuscFv) phage display library as an in vitro biological tool [35-37]. The LasB-neutralizing HuscFvs should be tested further toward clinical application as an adjunctive therapeutics for $P$. aeruginosa infection.

\section{Materials and Methods}

\subsection{Preparation of Native LasB}

Pseudomonas aeruginosa strain PAO1 was cultured in $250 \mathrm{~mL}$ of Luria-Bertani (LB) broth at $37^{\circ} \mathrm{C}$ with shaking aeration for $18 \mathrm{~h}$. The cell-free culture supernatant was collected after centrifugation at $15,000 \times \mathrm{g}, 4^{\circ} \mathrm{C}, 30 \mathrm{~min}$. Purification of the native LasB (nLasB) from the culture supernatant was carried out using ammonium sulfate precipitation and DEAE-Sepharose column chromatography. In brief, the proteins in the culture supernatant were subjected to gradient ammonium sulfate precipitation. Firstly, $250 \mathrm{~mL}$ of the culture supernatant was added with $75 \mathrm{~g}$ of ammonium sulfate powder to yield $30 \%$ saturation. After the precipitate was removed by centrifugation at $15,000 \times g, 4{ }^{\circ} \mathrm{C}, 30 \mathrm{~min}, 200 \mathrm{~g}$ of ammonium sulfate powder was added to the supernatant to $80 \%$ saturation. The precipitate 
was collected by centrifugation as above, resuspended in $10 \mathrm{~mL}$ of $20 \mathrm{mM}$ Tris-HCl buffer, $\mathrm{pH} 8.5$, and filtered through a sterile $0.45 \mu \mathrm{m}$ syringe filter. The preparation was dialyzed against the same buffer using Amicon ${ }^{\circledR}$ Ultra $4 \mathrm{~mL} 3 \mathrm{~K}$ centrifugal filter devices (Merck Millipore, Darmstadt, Germany) and further purified by using DEAE-Sepharose Fast Flow resin (GE Healthcare Life Sciences, Sweden). The protein solution was loaded onto the DEAE column pre-equilibrated with $20 \mathrm{mM}$ Tris- $\mathrm{HCl}$, pH 8.5. After allowing the proteins to bind to the resin for $1 \mathrm{~h}$, the column was washed with the same buffer to remove the unbound proteins; then, the target enzyme was eluted by applying a linear gradient of $\mathrm{NaCl}(100-600 \mathrm{mM})$. Each collected fraction was analyzed by sodium dodecyl sulfatepolyacrylamide gel electrophoresis (SDS-PAGE) and Coomassie Brilliant Blue G-250 (CBB) staining. The fractions containing the $\mathrm{nLasB}$ were pooled and dialyzed against the same buffer. The protein quantity was determined by Bicinchoninic Acid Assay (BCA) using Pierce $^{\mathrm{TM}}$ BCA Protein Assay Kit (TermoFisher Scientific, Waltham, MA, USA) following the manufacturer's instruction. Different bovine serum albumin (BSA) concentrations were used for constructing the protein standard curve. Briefly, $25 \mu \mathrm{L}$ of each BSA standard solution (20-2000 $\mu \mathrm{g} / \mathrm{mL}$ ) and protein sample were prepared in microplate wells (triplicate). Then, $200 \mu \mathrm{L}$ of the working reagent (mixture of solutions $\mathrm{A}$ and B provided with the kit) were added to each well and mixed on a plate shaker. The plate was covered and incubated at $37{ }^{\circ} \mathrm{C}$ for $30 \mathrm{~min}$. Absorbance at $595 \mathrm{~nm}$ of the content in each well was determined spectrophotometrically. The optical densities (OD) $595 \mathrm{~nm}$ of the standard protein dilutions were plotted against the known protein concentrations to construct the standard curve. Protein concentration of the unknown sample was extrapolated from the standard curve. The preparation was verified by LC/MS-MS and stored at $-20{ }^{\circ} \mathrm{C}$ in small portions until use.

\subsection{Determination of Enzymatic Activity of $n L a s B$}

The elastase activity of the purified nLasB was determined by the fluorogenic substrate assay using EnzChek Elastase Assay Kit (Invitrogen, Carlsbad, CA, USA), following the manufacturer's instruction. The EnzChek Elastase Assay Kit provides a sensitive, convenient, and fast fluorometric method for measuring elastase (or other protease) activity or for screening the enzyme inhibitors in a high-throughput format. The substrate in this EnzChek kit is the highly fluorescein-labeled (BODIPY-FL-labeled) DQ-elastin conjugate where the fluorescence signal is quenched until enzymatic digestion to yield highly fluorescent fragments. The intensity of the emitted fluorescence signals correlates with the enzymatic activity. In the presence of the enzyme inhibitor, the fluorescence emission is reduced. For determining enzymatic activity of the $\mathrm{nLasB}, 25 \mu \mathrm{g} / \mathrm{mL}$ of the BODIPY FL-DQ conjugatedelastin substrate solution was added into individual wells of a microplate containing reaction buffer (provided with the kit). Different concentrations $(25,50,75$, and $100 \mathrm{nM})$ of nLasB were added appropriately into the wells, and fluorescence intensity of the content in each well was monitored using a fluorometer $(E x / E m=485 \pm 10 \mathrm{~nm} / 530 \pm 15 \mathrm{~nm})$ at $37^{\circ} \mathrm{C}$ for $2 \mathrm{~h}$. Porcine pancreatic elastase, provided with the assay kit, was used as a positive control.

The elastolytic activity of the nLasB was also determined by elastin Congo Red assay [13]. The basis of this assay is that digestion of elastin substrate (Congo Red-elastin complex) by LasB/elastase allows the Congo Red dye to be hydrolyzed and released from the dye-elastin complex, where the amount of the released dye directly correlates with the enzyme activity. In the presence of the enzyme inhibitor, the amount of the released dye is reduced. Various concentrations $(12.5,25,50,100$, and $200 \mathrm{nM})$ of purified nLasB were added into wells of the reaction buffer ( $50 \mathrm{mM}$ Tris- $\mathrm{HCl}, \mathrm{pH} 7.5$, and $0.5 \mathrm{mM} \mathrm{CaCl}_{2}$ ), containing $10 \mathrm{mg}$ of Congo Red-elastin substrate (Sigma, St. Louis, MO, USA). The reaction mixtures were incubated at $37{ }^{\circ} \mathrm{C}$ for $2 \mathrm{~h}$ and terminated by adding EDTA. The clear supernatant after pelleting the undigested substrate by centrifugation was transferred into a new tube and optical density (OD) at $495 \mathrm{~nm}$ was measured. 


\subsection{Phage Bio-Panning and Characterization of nLasB-Bound HuscFvs}

The human $\mathrm{scFv}$ (HuscFv) phage display library used in this study was constructed previously [37]. Total RNA were extracted from lymphocytes of 60 healthy young adult volunteers and reverse transcribed to cDNAs. The cDNAs were used as templates for PCR amplification of immunoglobulin genes coding for variable heavy chain (VH) domains $(v h)$ and variable kappa light chain (VL) domains $(v l)$ by error prone PCR using human degenerate primers designed from all families/subfamilies of the human immunoglobulin genes. After amplification, the $v h$ and $v l$ sequences were linked randomly [via a nucleotide linker coding for a peptide $\left(\mathrm{Gly}_{4} \mathrm{Ser}\right)_{3}$ ] into vh-linker-vl (huscfos) sequences before ligating to PCANTAB 5E phagemid vector downstream of the gene coding for a phage coat protein, P3. The recombinant huscfos-phagemids were used to infect competent TG1 E. coli bacteria. The huscfo-phagemid-transfected-E. coli were grown and co-infected with a helper phage (M13KO7). The complete phage particles displaying HuscFvs of variable specificities as fusion proteins with the phage P3 on their surface and carrying integrated huscfos in their genomes, were obtained from the bacterial culture supernatant. The HuscFv diversity (different epitope/antigen specificity) of this library was approximately $2.6 \times 10^{8}[37,38]$.

Phage clones displaying nLasB-bound HuscFvs were selected from the HuscFv phage display library by phage bio-panning process [37]. Briefly, $0.5 \mu \mathrm{g}$ of purified nLasB in $100 \mu \mathrm{L}$ of $0.2 \mathrm{M}$ sodium carbonate-bicarbonate buffer, $\mathrm{pH}$ 9.4, was added into a well of an EIA/RIA strip (Corning, NY, USA). After blocking the free spaces of the nLasB-coated well with Pierce $^{\mathrm{TM}}$ Protein-Free Blocking Buffer (ThermoFisher Scientific, Waltham, MA, USA), the HuscFv phage display library $(50 \mu \mathrm{L})$ was added into the well and incubated. The unbound phages were washed away; the nLasB-bound phages were used to infect HB2151 E. coli. Then, the infected bacteria were spread onto selective $2 \times$ YT agar plates supplemented with $100 \mu \mathrm{g} / \mathrm{mL}$ ampicillin and $2 \%(\mathrm{w} / \mathrm{v})$ glucose $\left(2 \mathrm{x}\right.$ YT-AG), and incubated at $37^{\circ} \mathrm{C}$ overnight. The phagemid-transformed HB2151 E. coli colonies grown on the agar were screened for HuscFv genes (huscfus) by direct colony PCR using PCANTAB-5E phagemidspecific primers [38]. The huscfv-positive E. coli clones were grown in $2 \times$ YT-AG broth at $37^{\circ} \mathrm{C}$ until the OD $600 \mathrm{~nm}$ reached $\sim 0.6$ (about $3 \mathrm{~h}$ ). The culture was added with $1 \mathrm{mM}$ isopropyl- $\beta$-D-1-thiogalactopyranoside (IPTG) (Thermo Fisher Scientific) to induce the recombinant HuscFv expression and the preparation was incubated further at $30{ }^{\circ} \mathrm{C}$ for 5-6 h. Bacterial cells were harvested by centrifugation, resuspended in PBS, sonicated and centrifuged to remove the cell debris. The expressed soluble HuscFvs in the lysates of the phage-transformed HB2151 E. coli were tested for their binding to nLasB by indirect ELISA [38]. The diversity of the nucleotide sequences of the huscfos was determined by Sanger sequencing. The huscfo sequences coding for soluble nLasB-bound HuscFvs of individual HB2151 E. coli clones were deduced, and their canonical complementaritydetermining regions (CDRs) and immunoglobulin framework regions (FRs) of the $\mathrm{VH}$ and VL domains as well as the inter-domain linker peptides were worked out, based on the numbering scheme of Chotia and Kobat [39].

\subsection{Large Scale Production of Recombinant LasB-Bound HuscFvs}

For large scale production of the nLasB-bound HuscFvs, the pCANTAB-5E phagemids harboring huscfos were sub-cloned individually into the pLATE52 expression vector (Thermo Fisher Scientific) using the ligation-independent cloning (LIC) protocol (aLICator LIC Cloning and Expression Kit 4; Thermo Fisher Scientific). The recombinant pLATE52huscfo plasmids were transformed into JM109 E. coli. After colony PCR screening for the presence of huscfos and DNA sequencing (Sanger) for the gene verification, the verified recombinant plasmids were introduced into NiCo21(DE3) E. coli (New England Biolabs, St. Albans, UK). A single colony of each transformed NiCo21(DE3) E. coli clone was cultured in $1 \mathrm{mM}$ IPTG-conditioned broth to express recombinant HuscFvs. The recombinant HuscFvs were purified from the bacterial inclusion bodies (IBs) and refolded as previously described [40]. The concentrations of the refolded antibody preparations were determined using Pierce ${ }^{\circledR}$ BCA Protein Assay Kit (Thermo Fisher Scientific). The quality and purity 
of the recombinant antibodies were analyzed by SDS-PAGE and CBB staining. The buffer of the refolded HuscFv preparations was changed to $20 \mathrm{mM}$ Tris- $\mathrm{HCl}, \mathrm{pH} 8.5$, by dialysis; then, the preparations were concentrated using Amicon ${ }^{\circledR}$ Ultra $4 \mathrm{~mL} 3 \mathrm{~K}$ centrifugal filter devices (Merck Millipore), filtered through a $0.2 \mu \mathrm{m}$ low-protein-binding Acrodisc ${ }^{\circledR}$ syringe filter (Pall, Port Washington, NY, USA), and kept in $8 \%(w / v)$ glycerol at $-80^{\circ} \mathrm{C}$ for further use.

The HuscFvs were subjected to circular dichroism (CD) analysis for determining their secondary structure and retested for nLasB binding by indirect ELISA to ensure their proper folding (retained specificity of the soluble HuscFv counterparts from the HB2151 E. coli).

For the CD analysis, the HuscFvs buffer was changed to $20 \mathrm{mM}$ sodium phosphate buffer, $\mathrm{pH} 8.5$, by dialysis and the protein concentration was adjusted to $0.1 \mathrm{mg} / \mathrm{mL}$. The secondary structure of the refolded LasB-bound HuscFvs was determined using far-UV CD. The CD analysis was carried out on a JASCO J-815 spectropolarimeter equipped with a Peltier temperature controller system (Jasco, Tokyo, Japan). The CD spectra (190-260 nm) of refolded antibodies were recorded in a 1-mm path-length quartz cuvette at $25^{\circ} \mathrm{C}$ and a scan rate of $50 \mathrm{~nm} / \mathrm{min}$. Three scans were averaged to generate the CD spectrum for each protein.

\subsection{Fluorogenic Substrate Assay for Determining the LasB Neutralizing Activity of the HuscFvs}

The inhibitory effect of HuscFvs on the elastolytic (elastase) activity of the nLasB was assessed using fluorogenic substrate (EnzChek elastase assay kit; Invitrogen), following the manufacturer's instruction. The elastase inhibition assay was performed by preparing the reaction mixtures (each mixture in triplicate): HuscFvs $(2.5,5,7.5$, and $10 \mu \mathrm{M})$ mixed with $25 \mathrm{nM}$ nLasB (test mixtures), $25 \mathrm{nM}$ nLasB mixed with $5 \mathrm{mM}$ EDTA (positive inhibition control mixture), and $25 \mathrm{nM}$ nLasB in buffer (negative inhibition control). All preparations were kept at room temperature away from the light for $15 \mathrm{~min}$. Fifty microliters of $25 \mu \mathrm{g} / \mathrm{mL}$ the BODIPY-FL-labeled DQ-elastin substrate solution was added to each mixture and kept at room temperature in the dark for $30 \mathrm{~min}$. The fluorescence emission was measured at $515 \mathrm{~nm}$ with excitation at $505 \mathrm{~nm}$ using a fluorescent microplate reader (Synergy H1 Hybrid Reader; BioTek, Winooski, VT, USA). The fluorescence signal increased after the substrate was digested by the active LasB (elastase). The signal is reduced in the presence of the enzyme inhibitor, compared to the reaction without the inhibitor. Three independent experiments were performed.

\subsection{Elastin Congo Red Assay for Determining the HuscFvs-Mediated Inhibition of the LasB Elastolytic Activity}

HuscFvs-mediated neutralization of the nLasB elastolytic activity was evaluated also by the elastin Congo Red assay. The purified nLasB at $50 \mathrm{nM}(100 \mu \mathrm{L})$ was mixed with various concentrations of HuscFvs $(0.625,1.25,2.5$, and $5 \mu \mathrm{M})$. Each mixture was incubated at $37{ }^{\circ} \mathrm{C}$ for $1 \mathrm{~h}$. LasB alone served as negative neutralization control and $5 \mathrm{mM}$ EDTA as positive neutralization control. Then the Congo Red-elastin substrate solution in reaction buffer was added and further incubated at $37^{\circ} \mathrm{C}$ for $2 \mathrm{~h}$ with shaking at $250 \mathrm{rpm}$. The reaction was stopped by adding $100 \mu \mathrm{L}$ of $0.12 \mathrm{M}$ EDTA. The clear supernatant was collected by centrifugation at $15,000 \times g$ for 25 min and transferred to a new tube. Hydrolyzed Congo Red was determined by measuring the absorbance of the solution at $495 \mathrm{~nm}$. Three independent experiments were performed. The net OD $495 \mathrm{~nm}$ of the each assay supernatant was obtained after subtracting with the background absorbance (OD $495 \mathrm{~nm}$ of the reaction mixture containing the indicated amount of HuscFv, substrate, and buffer without adding the nLasB).

\subsection{Homology Modeling and Inter-Molecular Docking}

The three-dimensional (3D) structure of wild-type P. aeruginosa elastase (PDB ID: 1EZM) was retrieved from the Protein Data Bank (RCSB PDB) [41]. The amino acid sequences of the HuscFvs were subjected to structural modeling using I-TASSER server 
service (http:/ / zhanglab.ccmb.med.umic.edu/I-TASSER/; accessed on 12 September 2020) $[42,43]$. The qualities of the I-TASSER-predicted 3D structural models were subsequently refined in order to improve the local geometric and physical quality of the predicted 3D structure using ModRefiner high-resolution protein structure refinement (http:/ / zhanglab.ccmb.med.umich.edu/ModRefiner / ; accessed on 15 September 2020) [44], and fragment-guided molecular dynamics (FG-MD) simulation (http:/ / zhanglab.ccmb. med.umich.edu/FG-MD/; accessed on 16 September 2020) [45]. The refined HuscFv 3D models and LasB 3D structure were subjected to intermolecular docking using the automated ClusPro 2.0 protein-antibody docking server [46-48]. All antigen-antibody complexed models were analyzed and visualized using Discovery Studio 3.5 and PyMol software (PyMol Molecular Graphics System, Version 2 edu, Schrodinger, LLC).

\subsection{Statistical Analysis}

GraphPad Prism 5 software (GraphPad, La Jolla, CA, USA) was used to compare the results of all tests. Statistically significant differences between groups were determined by one-way ANOVA and Tukey's post hoc multiple comparison tests. All data are presented as mean \pm SD. $p$-value $<0.05$ was considered statistically significant.

\section{Results}

\subsection{Purification and Characterization of the $n L a s B$}

The nLasB was eluted from the DEAE-Sepharose Fast Flow column at $300 \mathrm{mM} \mathrm{NaCl}$. The purified nLasB protein after SDS-PAGE and CBB staining appear as predominant band at apparent molecular weight $33 \mathrm{kDa}$ (Figure 1A). The 33-kDa band and the faint 55-kDa band in the SDS-PAGE gel were verified by LC-MS/MS as the P. aeruginosa elastase and immunomodulatory metalloprotease, respectively (Supplementary Table S1).

\subsection{Enzymatic Activities of the Native LasB}

The enzyme activity of the purified nLasB was determined using EnzChek ${ }^{\mathrm{TM}}$ elastase assay. The fluorescence intensity increased with the increasing amounts of the nLasB (Figure 1B).

Figure $1 \mathrm{C}$ shows results of the elastin Congo Red assay for testing elastolytic activity of the nLasB. The results are conformed to those of the fluorogenic substrate assay. The absorbance at $495 \mathrm{~nm}$ (Congo Red released from digested substrate) increased in nLasB dose-dependent manner. The results of both assays indicated that the so-prepared purified nLasB retained the inherent elastolytic activities. Therefore, the enzymatically active nLasB was used as antigen for phage bio-panning for selecting HuscFv-displaying phage clones that bound to the $\mathrm{nLasB}$ and for studying the elastase neutralizing activity of the nLasB-bound HuscFvs.

\subsection{Selection and Characterization of LasB-Bound HuscFv-Displaying Phages}

After single-round bio-panning with the active nLasB protein, a total of 118 phagetransformed E. coli clones that grew on the selective agar plates were subjected to colony PCR analysis for determining the HuscFv-coding genes (huscfus). Among these clones, 45 clones $(38.13 \%)$ were huscfv-positive as they yielded 1000-bp amplicons, indicating that they carried recombinant huscfo-phagemids. Representatives of the huscfv-positive clones (clones 3-5, 7, and 10) are shown in Figure 1D. The other clones (1, 2, 6, 8, and 9) in the same Figure carried truncated huscfo sequences.

After the huscfo-positive HB2151, E. coli were grown under IPTG induction, lysates of 21 huscfv-positive clones could express soluble HuscFv proteins. By indirect ELISA, the HuscFvs of two phage-transformed E. coli clones (nos. N42 and N45) gave significant ELISA signals to nLasB (more than two times to the antigen control or BSA) and above the background control (HB2151, lysate of original HB2151 E. coli without huscfv-phagemid) (Figure 1E). The huscfos of these two clones showed complete DNA sequences coding for 
VH-linker-VL (HuscFvs). Therefore, the huscfos of the N42 and N45 E. coli clones were sub-cloned into plasmid vector for large-scale production of the HuscFvs.

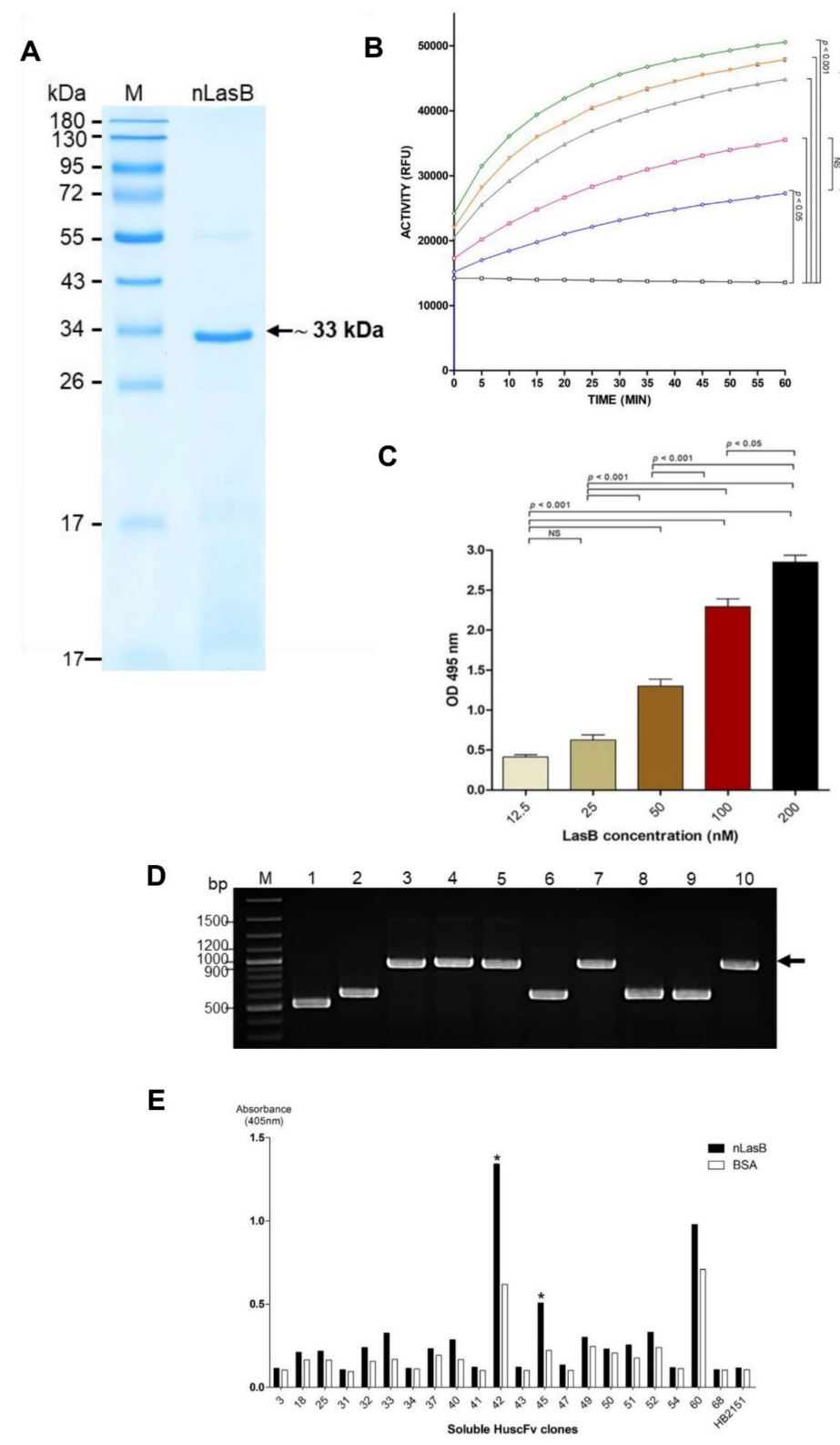

Figure 1. Preparation of native LasB (nLasB) and selection of the LasB-bound HuscFv-displaying phage clones. (A) Purified nLasB revealed by SDS-PAGE and CBB staining. M, protein molecular mass marker (ThermoFisher Scientific, Rockford, $1 \mathrm{~L}$, USA); nLasB, purified native LasB ( $\sim 33 \mathrm{kDa}$, arrow). Numbers at the left are protein masses in $\mathrm{kDa}$. (B) Determination of enzymatic activities of the purified nLasB using fluorogenic substrate assay. PC, positive control which was porcine pancreatic elastase $(0.025 \mathrm{U} / \mathrm{mL})$ mixed with substrate; NC, negative control which was reaction buffer mixed with substrate; ACTIVITY (RFU), relative fluorescence units of the enzymatic activity per minute. (C) Results of elastin-Congo Red assay for determining elastolytic activity of the nLasB. NS, not significantly different. (D) Colony-PCR analysis of representative phage-transformed HB2151 E. coli clones; black arrow indicates amplicon of complete huscfus (1000 bp) in the phage-transformed-E. coli. Smaller DNA bands are truncated huscfus. M, DNA marker (Thermo Fisher Scientific); 1-10, the phage-transformed E. coli clones nos. 1-10, respectively. Numbers at the left are DNA masses in base pairs (bp). (E) Results of indirect ELISA for determining the binding of HuscFvs in lysates of phage-transformed HB2151 E. coli clones to nLasB, control BSA (antigen control), and HB2151 (lysate of original HB2151 E. coli without huscfv-phagemid) as background binding control; asterisks indicate the clones that their HuscFvs gave the high binding activity to nLasB (an ELISA signal at OD $405 \mathrm{~nm}$ to the nLasB: OD $405 \mathrm{~nm}$ to the control BSA was more than 2). These clones were selected for further experiments. 


\subsection{Recombinant HuscFvs to LasB}

After subcloning of the huscfus of the E. coli clones N42 and N45 from recombinant pCANTAB-5E phagemids into the pLATE52 plasmids, the PCR products showed the bands at the expected size of around $850 \mathrm{bp}$ (Figure 2A). The LasB-bound HuscFvs with the $6 \times$ His tag at the $\mathrm{N}$-terminal and E-tag at the $\mathrm{C}$-terminal were expressed from the huscfo-plasmid transformed NiCo21(DE3) E. coli under IPTG induction. Figure 2B shows SDS-PAGE-separated- and CBB-stained LasB-bound HuscFvs $(\sim 30,35 \mathrm{kDa})$ after purification and refolding from the inclusion bodies of the N42 and N45 E. coli clones. From the program analysis of the CD spectra (Figure 2C), the refolded HuscFv-N42 and HuscFv-N45 were found to acquire mainly $\beta$-sheet structure, which is the characteristic of immunoglobulin molecule. Moreover, the refolded HuscFvs retained the nLasB binding ability, as demonstrated by indirect ELISA (Supplementary Figure S1), indicating correct folding of the antibody molecules.
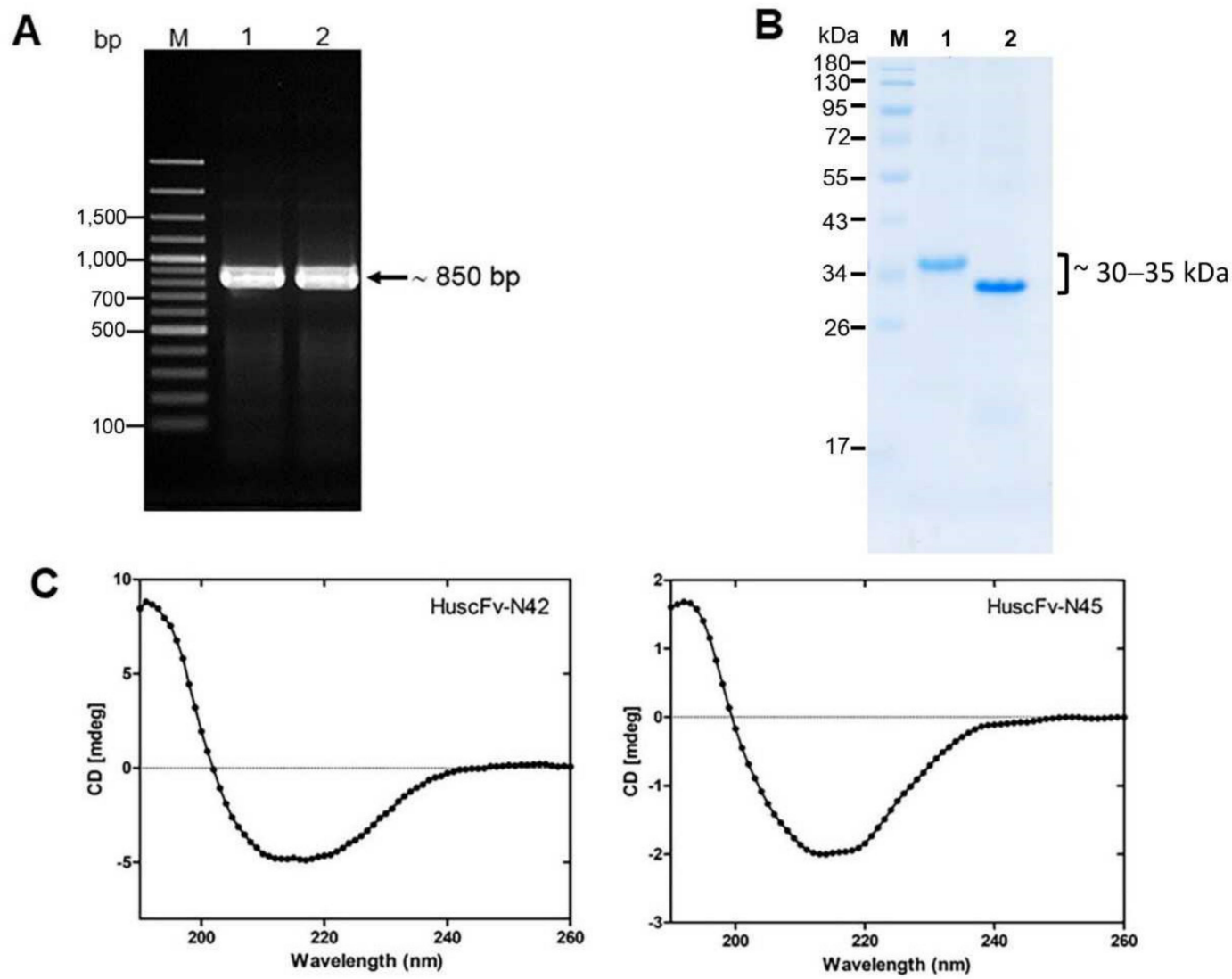

Figure 2. Production of recombinant LasB-bound HuscFvs. (A) Amplicons of huscfv-LIC fragments for sub-cloning into pLATE52 vector. M, 100-bp plus DNA marker; 1 and 2, huscfo-LIC amplicons of the transformed NiCo21(DE3) E. coli clones N42 and N45, respectively. Numbers at the left are DNA sizes in bp. (B) SDS-PAGE analysis of LasB-bound HuscFvs. M, protein marker; 1 and 2, purified HuscFv-N42 and HuscFv-N45, respectively ( 30, $35 \mathrm{kDa})$. Numbers at the left are protein molecular masses in kDa. (C) CD spectra of the refolded HuscFv-N42 (left) and HuscFv-N45 (right).

\subsection{HuscFvs-Mediated Neutralization of the LasB Elastase Activity}

The fluorogenic substrate assay was used to determine the neutralizing activity of the HuscFvs on the LasB elastolytic activity. The percentages of relative fluorescence units emitted from the DO-elastin substrate after the enzyme was treated with different concentrations of HuscFvs and controls are shown in Figure 3A,B. The fluorescence intensity of nLasB treated with HuscFv-N42 showed decreasing trend as the antibody concentrations increased, albeit not significantly different from the nLasB alone (without HuscFvs). The nLasB treated with the HuscFv-N45 had significantly decreased enzymatic activity as 
the HuscFv concentrations increased. The results of this assay indicate that the HuscFvs neutralized the nLasB enzymatic activity in a dose-dependent manner. In this experiment, the $5 \mathrm{mM}$ EDTA which was used as positive LasB elastase neutralization control, effectively neutralized (abolished) the enzymatic activity.

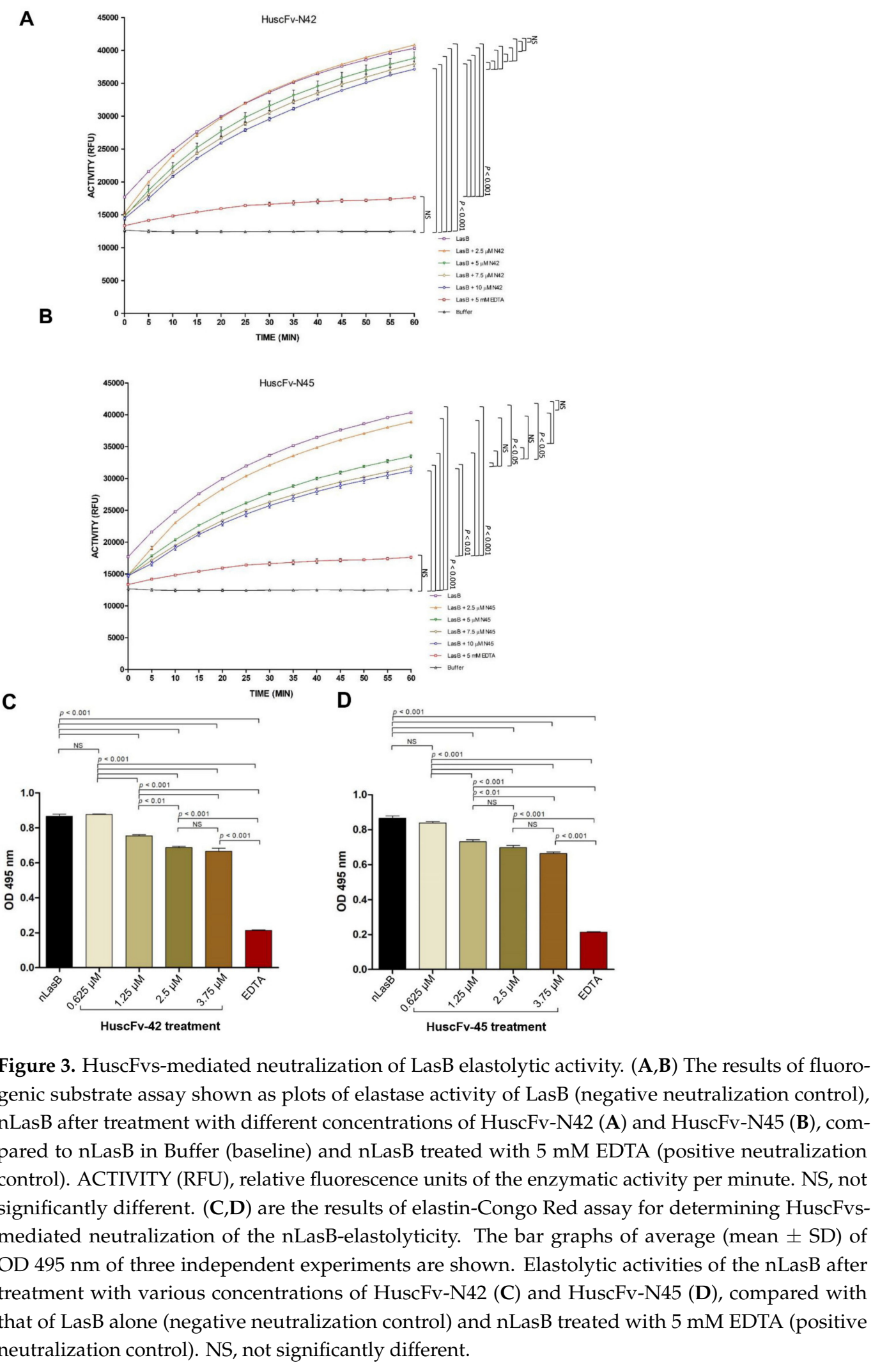


By using elastin Congo Red assay, there was a significant suppression of LasB elastase activity after HuscFvs-treatment (decreased OD $495 \mathrm{~nm}$ of the released Congo Red from the Congo Red-elastin substrate), compared the negative neutralization control (native LasB alone) (Figure 3C,D). The suppression also showed the HuscFv concentration-dependent trend similar to the fluorogenic substrate assay. These results indicate that the HuscFv-N42 and HuscFv-N45 have inhibitory ability on the nLasB elastolytic activity.

\subsection{Predicted LasB Residues Bound by HuscFvs}

The 3D models of the HuscFv-N42 and HuscFv-N45 showed reliable Ramachandran plots (Supplementary Figure S2). The proportions of residues in the most favored regions, the additional allowed regions, the generously allowed regions, and the disallowed regions of the Ramachandran diagrams of HuscFv-N42 were 87.3, 10.8, 0.0, and 2.0\% and those of the HuscFv-N45 were 90.2, 6.7, 1.5, and 1.5\%, respectively.

The lowest local energies of the interactions between Pseudomonas aeruginosa LasB (elastase) and HuscFv-N42 and HuscFv-N45, based on the ClusPro docking server were -17.4 and $-17.0 \mathrm{kcal} / \mathrm{mol}$, respectively. Details of the LasB-HuscFv intermolecular docking including the interactive residues of the LasB and the HuscFv amino acids and domains as well as the intermolecular bonds between the two parties are shown in Table 1 and Figure 4.

By the in silico docking, HuscFv-N42 was predicted to use VH-CDRs 2 and 3 and VL-CDRs 1-3, as well as the help from the peptide linker between the VH and VL domains, to form contact interfaces with the residues on the LasB target (right panel of Figure 4A and Table 1). There were 10 hydrogen bonds formed between the HuscFv-N42 and the LasB including S168 of VL-CDR1 with LasB D48; Y59 of VH-CDR2 with LasB E111; N232 of VL-CDR3 with LasB Y114; T57 of VH-CDR2 and R106 of VH-CDR3 with LasB T127; K190 of VL-CDR2 with LasB Y155; Q102 of VH-CDR3 with LasB R208; and S140 of the peptide linker with LasB S45, T46, and D47. Two salt bridge interactions were also demonstrated between HuscFv-N42 and the LasB including D62 of VH-CDR2 with LasB R108; and K190 of VL-CDR2 with LasB D221. A hydrophobic interaction ( $\pi-\pi$ stacking) between W103 of VH-CDR3 and H223 of the bacterial enzyme are also shown.

The HuscFv-N45 was predicted to form an ionic bond with D221 of the LasB protease through K58 of VH-CDR2 and R72 of VH-FR3. The antibody also used VH-CDR2, VH-FR1, and VH-FR3 to form hydrogen bonds with the target antigen, i.e., K13 of VH-FR1 with LasB T97 and Q149; S7, R19, and S21 of VH-FR1 with LasB W115; K58 of VH-CDR2 with LasB R156 and I220; Y60 of VH-CDR2 with LasB R156; and K76 of VH-FR1 with LasB H223. Lysine 76 of VH-FR1 and Y80 of VH-FR3 formed a contact with LasB E141 via salt bridge interaction. The salt bridge bonding also occurred between R19 of VH-FR1 and E164 of the LasB (right panel of Figure 4B).

Table 1. Presumptive residues of $P$. aeruginosa LasB (elastase) that were predicted by computer simulation to form the contact interface with residues and domains of the effective human singlechain antibodies, HuscFv-N42 and HuscFv-N45.

\begin{tabular}{cccc}
\hline LasB Protein & \multicolumn{2}{c}{ HuscFv-N42 } & Interactive Bond (s) \\
\cline { 1 - 3 } Residue & Residue & Domain (s) & Hydrogen \\
S45 & S140 & Linker & Hydrogen \\
T46 & S140 & Linker & Hydrogen \\
D47 & S140 & Linker & Hydrogen \\
D48 & S168 & VL-CDR1 & Salt bridge \\
R108 & D62 & VH-CDR2 & Hydrogen \\
E111 & Y59 & VH-CDR2 & Hydrogen \\
Y114 & N232 & VL-CDR3 & Hydrogen \\
T127 & T57/R106 & VH-CDR2/CDR3 & Hydrogen \\
R208 & K190 & VL-CDR2 & Hydrogen \\
Y155 (substrate binding) & Q102 & VH-CDR3 & Salt bridge \\
D221 (substrate binding) & K190 & VL-CDR2 & Hydrophobic $(\pi-\pi$ stacking) \\
\hline
\end{tabular}


Table 1. Cont.

\begin{tabular}{cccc}
\hline LasB Protein & \multicolumn{2}{c}{ HuscFv-N45 } & \multirow{2}{*}{ Interactive Bond(s) } \\
\hline Residue & Residue & Domain(s) & \\
\hline T97 & K13 & VH-FR1 & Hydrogen \\
W115 & S7/R19/S21 & VH-FR1 & Hydrogen \\
E141 (located at the center of the & K76/Y80 & VH-FR1/FR3 & Salt bridge \\
catalytic site) & K13 & VH-FR1 & Hydrogen \\
Q149 & K58/Y60 & VH-CDR2 & Hydrogen \\
R156 & R19 & VH-FR1 & Salt Bridge \\
E164 (ligand of zinc co-factor) & K58 & VH-CDR2 & Hydrogen \\
I220 & K58/R72 & VH-CDR2/FR3 & Ionic \\
D221 (substrate binding) & K76 & VH-FR3 & Hydrogen \\
H223 (substrate binding) & \multicolumn{2}{c}{}
\end{tabular}
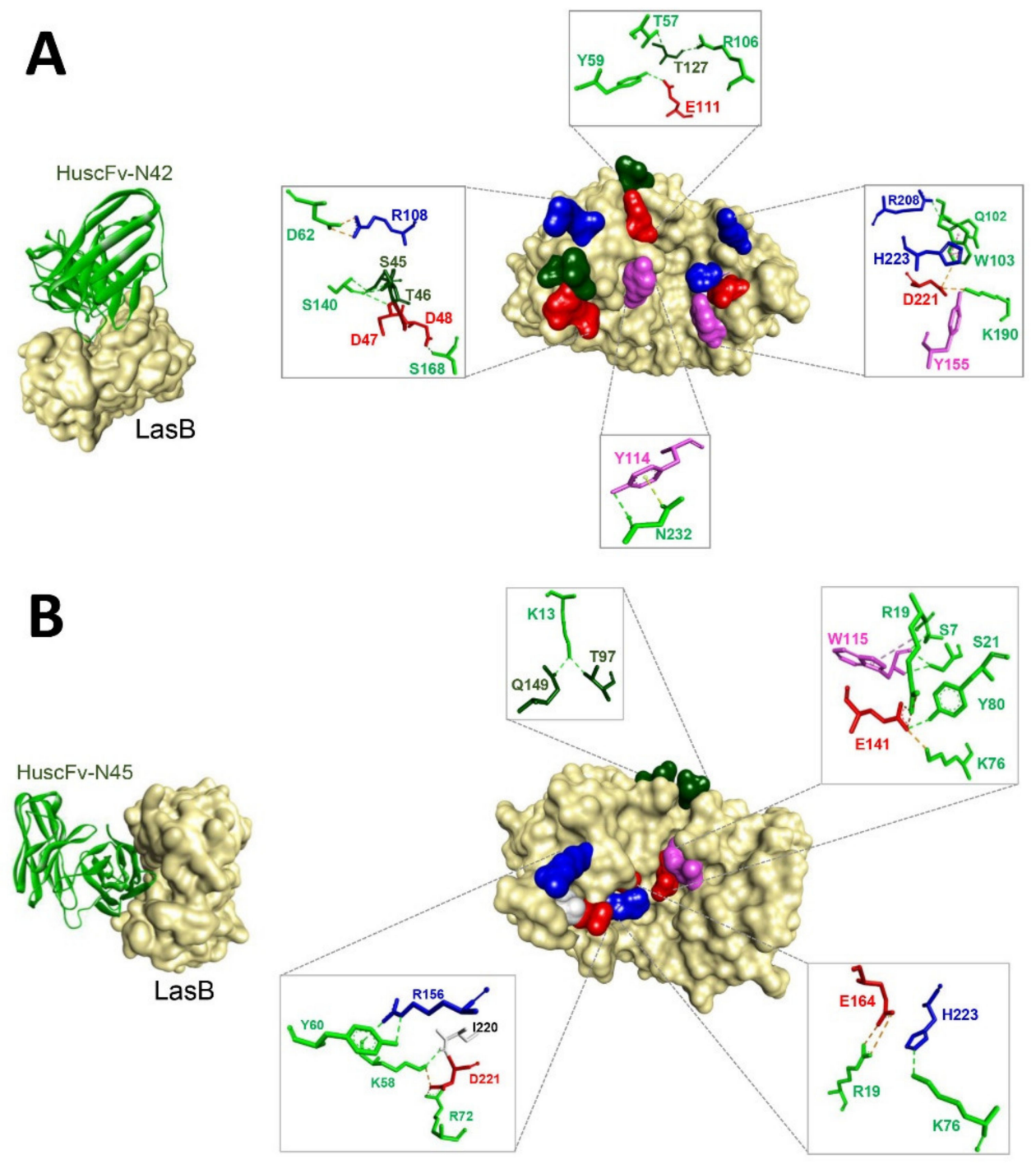

Figure 4. Presumptive binding of HuscFvs to LasB as predicted by computerized homology modeling and intermolecular docking. (A) LasB-HuscFv-N42 and (B) LasB-HuscFv-N45. Left panels of $(\mathbf{A}, \mathbf{B})$ show the presumptive interface contacts between the LasB (light brown) and the HuscFvs (green). Both antibodies interact with the LasB near or in the active/catalytic site. Right side of $(\mathbf{A}, \mathbf{B})$ shows contact residues of LasB with HuscFvs-N42 and HuscFv-N45, respectively. The LasB amino acids are colored according to the CINEMA color scheme: polar negative D and E, red; polar positive $\mathrm{H}$ and $\mathrm{R}$, blue; polar neutral $\mathrm{S}, \mathrm{T}$, and $\mathrm{Q}$, dark green; non-polar aromatic $\mathrm{W}$ and $\mathrm{Y}$, magenta; and non-polar aliphatic I, white (grey in B, right panel). 


\section{Discussion}

LasB (elastase/pseudolysin) is one of the extracellular proteases secreted by P. aeruginosa in the infected host and in in vitro culture. This detrimental enzyme has multifunctional activities; it degrades a variety of the host proteins in tissues, extracellular matrices, as well as the host immune components for bacterial thriving, successful establishment of the infection, dissemination, and invasion [2]. Thus, it is an attractive target of innovative therapeutic agents for disarming the infecting P. aeruginosa. The lasB is encoded by las $\mathrm{B}$ gene and is produced by the bacteria (both planktonic and biofilm forming cells) as an enzymatically inactive pre-proenzyme $(\sim 55 \mathrm{kDa})$ with a classical signal peptide $(2.4-\mathrm{kDa})$ and a covalently linked amino-terminal propeptide $(18-\mathrm{kDa})[2,49,50]$. The signal sequence is removed upon passage through the inner membrane into the periplasm, where the propeptide is rapidly cleaved off by autoproteolysis [51]. The propeptide also functions as an intramolecular chaperone required for correct folding and the LasB secretion competence $[52,53]$. The LasB translocates through the outer membrane by general secretory pathway (type II secretion system) into the bacterial milieu/culture medium as a 33-kDa mature and active protease [4]. In this study, the mature LasB was isolated from the culture supernatant of $P$. aeruginosa. The purified LasB preparation contained predominantly the $33-\mathrm{kDa}$ mature protein, with a trace of the pre-proenzyme $(55-\mathrm{kDa})$ and the $18-\mathrm{kDa}$ amino-terminal propeptide (Figure 1A); the two latter should be from the lytic bacterial cells in the culture. By using fluorescent substrate and Congo Red assays, the purified nLasB showed enzymatic activity of which the kinetics of substrate cleavage determined by the former assay are, more or less, similar to those reported previously [54].

The active LasB/elastin was used as a bait (antigen) to fish out the HuscFv-dispalying phage clones from the previously constructed HuscFv phage display library by means of the conventional phage bio-panning process. Soluble HuscFvs recovered from the lysates of two huscfv-phagemid-infected HB2151 E. coli clones bound to the purified nLasB. Since the expression of the soluble HuscFvs from the huscfv-phagemids by the HB2151 E. coli is under a weak promotor ( $\mathrm{pLacZ}$ ), the amounts of the required HuscFvs were inadequate for further experiments. We, therefore, subcloned the huscfos into pLATE52 expression plasmid and used the NiCo21(DE3) E. coli as the factory for large scale production of the HuscFvs. The huscfv- pLATE52 transformed E. coli produced high amount of the HuscFvs in the bacterial inclusion bodies; thus, purification and refolding of the antibodies had to be performed. After refolding of the recombinant antibodies, we checked them by circular dichroism and found that the proteins had high percentages of $\beta$-sheet structure, indicating that they were likely immunoglobulins. The purified refolded proteins showed molecular masses at $\sim 30$ and $\sim 35 \mathrm{kDa}$ (Figure $2 \mathrm{~B}$ ), which are the correct sizes of the single-chain antibodies that consists of $\mathrm{VH}-\left(\mathrm{Gly}_{4} \mathrm{Ser}\right)_{3}-\mathrm{VL}$. Besides, the refolded proteins bound to the nLasB as did the soluble HuscFvs derived from the huscfv-phagemid-transformed HB2151 E. coli clones N42 and N45, verifying that they are nLasB-bound HuscFvs. We, therefore, tested the HuscFv-N42 and HuscFv-N45 further for their ability to neutralize the elastrolytic activity of the nLasB using the EDTA as the positive inhibitor control.

The HuscFvs from both E. coli clones were found to neutralize the nLAsB enzymatic activity to significant degrees in both in vitro assays (fluorescence substrate and Congo Red assays) in a dose-dependent manner. The HuscFv-N45 performed better than the HuscFv-N42 on the same concentration basis. The EDTA at $5 \mathrm{mM}$, which was used as positive $\mathrm{nLasB}$ neutralization control, effectively neutralized (abolished) the nLasB activity. Less amounts of the EDTA (in $\mu \mathrm{M}$ range) were not tested. There are possibilities to enhance the LasB neutralizing activity of the HuscFvs by either adjusting the antibody to target ratio in the in vitro assay such that the two reactants reach their equivalence zone. Moreover, target binding affinity of the antibodies may be increased by CDR resurfacing [55], i.e., point mutation of some residues exposed on the CDR surface without changing the ligand binding specificities and the CDR conformations. It should be noted that the HuscFvs from the N42 and N45 E. coli clones had different molecular masses although the lowest energy that they used to form contact interfaces with the LasB target were relatively similar ( -17.4 
and $-17.0 \mathrm{kcal} / \mathrm{mol}$, respectively) as predicted by the computerized molecular docking. The molecular mass difference should be from the different numbers and sizes of amino acid residues that the two HuscFvs contained especially at the VH CDR3 which is the main target binding domain of antibody. VH CDR3 lengths in human ranged from 4 to 36 residues according to the IMGT numbering; the average length of VH CDR3 in human is $15.5 \pm 3.2$ amino acid residues and the most frequently occurring length in human VH CDR3 was 14 residues [56]. The difference in the molecular contents which leads to different sizes and conformations of the HuscFvs-tertiary structures should explain the difference in target binding specificity, affinity, and/or functional efficacy of the different HuscFvs.

Although the detailed mechanisms of the HuscFvs in mediating the protease neutralization requires elucidation by laboratory experiments, the computerized simulation indicated that the HuscFv-N42 and HuscFv-N45 formed contact interfaces with residues critical for LasB-catalytic activities. The LasB residues bound by the HuscFv-N42, i.e., Y155, D221, and H223, are involved in substrate binding [41,57], while the HuscFv-N45 not only interacted with the substrate binding residues, D221 and H223, but also other amino acids, i.e., E164, which is the important ligand of zinc co-factor of this metalloproteinase [41], and the E141 located in the center of the elastase catalytic site, which is pivotal for the LasB protease activity [41,58]. The binding to different residues of the target should explain the better enzyme neutralizing ability of the HuscFv-N45 than the HuscFv-N42.

\section{Conclusions}

Engineered human monoclonal single-chain antibodies (HuscFvs) that neutralize the enzymatic activity of the $P$. aeruginosa LasB elastase were successfully generated using phage display technology. The HuscFvs should be tested further step-by-step towards the clinical use for mitigation of the disease severity caused by $P$. aeruginosa infection.

Supplementary Materials: The following data are available online https:/ / www.mdpi.com/article / 10.3390/pathogens10060765/s1, Figure S1: Ramachandran plots of the modeled HuscFv-N42 and HuscFv-N45; Table S1: LC-MS/MS of purified native LasB; Table S2: The predicted binding affinity $(\Delta \mathrm{G})$ and dissociation constant $(\mathrm{Kd})$ of LasB bound by individual HuscFvs.

Author Contributions: Conceptualization, N.I. and W.C.; methodology, N.I., W.C., S.S. and T.K.n.; software, S.S.; validation, M.C.-N., P.P. and N.S.; formal analysis, N.I., W.C., S.S. and T.K.-n.; investigation, S.S. and T.K.-n.; resources, N.I., W.C. and U.B.; writing-original draft preparation, S.S.; writing-review and editing, N.I., U.B. and W.C.; supervision, W.C., N.S., U.B., and N.I.; funding acquisition, N.I. and W.C. All authors have read and agreed to the published version of the manuscript.

Funding: This research was funded by the Thailand Research Fund (Grant no. RSA5980048) to N.I. and an NSTDA Chair Professor grant funded by the Crown Property Bureau of Thailand (Grant no. P-1450624) to W.C.

Institutional Review Board Statement: Not applicable.

Informed Consent Statement: Not applicable.

Data Availability Statement: The data used to support the findings of this study are available from the corresponding author upon request.

Acknowledgments: We acknowledge the Center of Research Excellence on Therapeutic Proteins and Antibody Engineering, Department of Parasitology, and Biomedical Research Unit, Department of Research, Faculty of Medicine Siriraj Hospital, for their technical support.

Conflicts of Interest: The authors declare no conflict of interest.

\section{References}

1. Morihara, K. Pseudolysin and other pathogen endopeptidases of thermolysin family. Methods Enzymol. 1995, $248,242-253$. [PubMed] 
2. Bever, R.A.; Iglewski, B.H. Molecular characterization and nucleotide sequence of the Pseudomonas aeruginosa elastase structural gene. J. Bacteriol. 1988, 170, 4309-4314. [CrossRef] [PubMed]

3. Braun, P.; de Groot, A.; Bitter, W.; Tommassen, J. Secretion of elastinolytic enzymes and their propeptides by Pseudomonas aeruginosa. J. Bacteriol. 1998, 180, 3467-3469. [CrossRef] [PubMed]

4. Kessler, E.; Safrin, M.; Gustin, J.K.; Ohman, D.E. Elastase and the LasA protease of Pseudomonas aeruginosa are secreted with their propeptides. J. Biol. Chem. 1998, 273, 30225-30231. [CrossRef]

5. Durand, É.; Bernadac, A.; Ball, G.; Lazdunski, A.; Sturgis, J.N.; Filloux, A. Type II protein secretion in Pseudomonas aeruginosa: The pseudopilus is a multifibrillar and adhesive structure. J. Bacteriol. 2003, 185, 2749-2758. [CrossRef]

6. Filloux, A. Protein secretion systems in Pseudomonas aeruginosa: An essay on diversity, evolution, and function. Front. Microbiol. 2011, 2, 155. [CrossRef]

7. Morihara, K.; Tsuzuki, H. Production of protease and elastase by Pseudomonas aeruginosa strains isolated from patients. Infect. Immun. 1977, 15, 679-685. [CrossRef]

8. Rawlings, N.D.; Barrett, A.J.; Bateman, A. MEROPS: The peptidase database. Nucleic Acids Res. 2010, 38, D227-D233. [CrossRef]

9. Morihara, K.; Tsuzuki, H.; Oka, T.; Inoue, H.; Ebata, M. Pseudomonas aeruginosa elastase: Isolation, crystallization, and preliminary characterization. J. Biol. Chem. 1965, 240, 3295-3304. [CrossRef]

10. Hersh, L.B.; Morihara, K. Comparison of the subsite specificity of the mammalian neutral endopeptidase 24.11 (enkephalinase) to the bacterial neutral endopeptidase thermolysin. J. Biol. Chem. 1986, 261, 6433-6437. [CrossRef]

11. Galloway, D.R. Pseudomonas aeruginosa elastase and elastolysis revisited: Recent developments. Mol. Microbiol. 1991, 5, $2315-2321$. [CrossRef]

12. Heck, L.W.; Morihara, K.; McRae, W.B.; Miller, E.J. Specific cleavage of human type III and IV collagens by Pseudomonas aeruginosa elastase. Infect. Immun. 1986, 51, 115-118. [CrossRef]

13. Kessler, E.; Safrin, M. Elastinolytic and proteolytic enzymes. In Pseudomonas Methods and Protocols; Humana Press: New York, NY, USA, 2014; pp. 135-169.

14. Döring, G.; Obernesser, H.J.; Botzenhart, K. Extracellular toxins of Pseudomonas aeruginosa. II. Effect of two proteases on human immunoglobulins IgG, IgA and secretory IgA. Zentralblatt Bakteriol. 1 Abt. Origin. A Med. Mikrobiol. Infekt. Parasitol. 1981, 249, 89-98.

15. Holder, I.A.; Wheeler, R. Experimental studies of the pathogenesis of infections owing to Pseudomonas aeruginosa: Elastase, an IgG protease. Can. J. Microbiol. 1984, 30, 1118-1124. [CrossRef]

16. Bastaert, F.; Kheir, S.; Saint-Criq, V.; Villeret, B.; Dang, P.M.C.; El-Benna, J.; Sirard, J.; Voulhoux, R.; Sallenave, J.M. Pseudomonas aeruginosa LasB subverts alveolar macrophage activity by interfering with bacterial killing through downregulation of innate immune defense, reactive oxygen species generation, and complement activation. Front. Immunol. 2018, 9, 1675. [CrossRef]

17. Mariencheck, W.I.; Alcorn, J.F.; Palmer, S.M.; Wright, J.R. Pseudomonas aeruginosa elastase degrades surfactant proteins A and D. Am. J. Respir. Cell. Mol. Biol. 2003, 28, 528-537. [CrossRef]

18. Komori, Y.; Nonogaki, T.; Nikai, T. Hemorrhagic activity and muscle damaging effect of Pseudomonas aeruginosa metalloproteinase (elastase). Toxicon 2001, 39, 1327-1332. [CrossRef]

19. Azghani, A.O.; Miller, E.J.; Peterson, B.T. Virulence factors from Pseudomonas aeruginosa increase lung epithelial permeability. Lung 2000, 178, 261-269. [CrossRef]

20. Vandivier, R.W.; Fadok, V.A.; Hoffmann, P.R.; Bratton, D.L.; Penvari, C.; Brown, K.K.; Brain, J.D.; Accurso, F.J.; Henson, P.M. Elastase-mediated phosphatidylserine receptor cleavage impairs apoptotic cell clearance in cystic fibrosis and bronchiectasis. J. Clin. Investig. 2002, 109, 661-670. [CrossRef]

21. Yanagihara, K.; Tomono, K.; Kaneko, Y.; Miyazaki, Y.; Tsukamoto, K.; Hirakata, Y.; Mukae, H. Role of elastase in a mouse model of chronic respiratory Pseudomonas aeruginosa infection that mimics diffuse panbronchiolitis. J. Med. Microbiol. 2003, 52, 531-535. [CrossRef]

22. Schmidtchen, A.; Holst, E.; Tapper, H.; Björck, L. Elastase-producing Pseudomonas aeruginosa degrade plasma proteins and extracellular products of human skin and fibroblasts, and inhibit fibroblast growth. Microb. Pathog. 2003, 34, 47-55. [CrossRef]

23. Hobden, J.A. Pseudomonas aeruginosa proteases and corneal virulence. DNA Cell Biol. 2002, 21, 391-396. [CrossRef]

24. Cathcart, G.R.; Quinn, D.; Greer, B.; Harriott, P.; Lynas, J.F.; Gilmore, B.F.; Walker, B. Novel inhibitors of the Pseudomonas aeruginosa virulence factor LasB: A potential therapeutic approach for the attenuation of virulence mechanisms in pseudomonal infection. Antimicrob. Agents Chemother. 2011, 55, 2670-2678. [CrossRef] [PubMed]

25. Zhu, J.; Cai, X.; Harris, T.L.; Gooyit, M.; Wood, M.; Lardy, M.; Janda, K.D. Disarming Pseudomonas aeruginosa virulence factor LasB by leveraging a Caenorhabditis elegans infection model. Chem. Biol. 2015, 22, 483-491. [CrossRef]

26. Sun, J.; LaRock, D.; Skowronski, E.; Kimmey, J.M.; Olson, J.; Jiang, Z.; O'Donoghue, A.J. Role of inflammasome-independent activation of IL-1 $\beta$ by the Pseudomonas aeruginosa protease LasB. bioRxiv 2020. [CrossRef]

27. Cowell, B.A.; Twining, S.S.; Hobden, J.A.; Kwong, M.S.; Fleiszig, S.M. Mutation of lasA and lasB reduces Pseudomonas aeruginosa invasion of epithelial cells. Microbiology 2003, 149, 2291-2299. [CrossRef]

28. Tang, H.B.; DiMango, E.; Bryan, R.; Gambello, M.; Iglewski, B.H.; Goldberg, J.B.; Prince, A. Contribution of specific Pseudomonas aeruginosa virulence factors to pathogenesis of pneumonia in a neonatal mouse model of infection. Infect. Immun. 1996, 64, 37-43. [CrossRef] 
29. Tan, M.W.; Rahme, L.G.; Sternberg, J.A.; Tompkins, R.G.; Ausubel, F.M. Pseudomonas aeruginosa killing of Caenorhabditis elegans used to identify P. aeruginosa virulence factors. Proc. Natl. Acad. Sci. USA 1999, 96, 2408-2413. [CrossRef]

30. Schimmel, P.; Tao, J.; Hill, J. Aminoacyl tRNA synthetases as targets for new anti-infectives. FASEB J. 1998, 12, 1599-1609. [CrossRef] [PubMed]

31. Bryskier, A. Antimicrobial Agents: Antibacterials and Antifungals; ASM Press: Washington, DC, USA, 2005; ISBN 1-55581-237-6.

32. Hu, Y.; Keniry, M.; Palmer, S.O.; Bullard, J.M. Discovery and analysis of natural-product compounds inhibiting protein synthesis in Pseudomonas aeruginosa. Antimicrob. Agents Chemother. 2016, 60, 4820. [CrossRef]

33. Galdino, A.C.M.; Viganor, L.; de Castro, A.A.; da Cunha, E.F.; Mello, T.P.; Mattos, L.M.; Pereira, M.D.; Hunt, M.C.; O'Shaughnessy, M.; Howe, O.; et al. Disarming Pseudomonas aeruginosa virulence by the inhibitory action of 1, 10-phenanthroline-5, 6-dione-based compounds: Elastase B (lasB) as a chemotherapeutic target. Front. Microbiol. 2019, 10, 1701. [CrossRef] [PubMed]

34. Adekoya, O.A.; Sjøli, S.; Wuxiuer, Y.; Bilto, I.; Marques, S.M.; Santos, M.A.; Nuti, E.; Cercignani, G.; Rossello, A.; Winberg, J.O.; et al. Inhibition of pseudolysin and thermolysin by hydroxamate-based MMP inhibitors. Eur. J. Med. Chem. 2015, 89, 340-348. [CrossRef] [PubMed]

35. Smith, G.P. Filamentous fusion phage: Novel expression vectors that display cloned antigens on the virion surface. Science 1985, 228, 1315-1317. [CrossRef] [PubMed]

36. Herrington-Symes, A.P.; Farys, M.; Khalili, H.; Brocchini, S. Antibody fragments: Prolonging circulation half-life special issueantibody research. Adv. Biosci. Biotechnol. 2013, 4, 689-698. [CrossRef]

37. Kulkeaw, K.; Sakolvaree, Y.; Srimanote, P.; Tongtawe, P.; Maneewatch, S.; Sookrung, N.; Tungtrongchitr, A.; Tapchaisri, P.; Kurazono, H.; Chaicumpa, W. Human monoclonal ScFv neutralize lethal Thai cobra, Naja kaouthia, neurotoxin. J. Proteom. 2009, 72, 270-282. [CrossRef]

38. Chaisri, U.; Chaicumpa, W. Evolution of therapeutic antibodies, influenza virus biology, influenza, and influenza immunotherapy. BioMed Res. Int. 2018, 2018, 1-23. [CrossRef]

39. Abhinandan, K.R.; Martin, A.C. Analysis and improvements to Kabat and structurally correct numbering of antibody variable domains. Mol. Immunol. 2008, 45, 3832-3839. [CrossRef]

40. Santajit, S.; Seesuay, W.; Mahasongkram, K.; Sookrung, N.; Pumirat, P.; Ampawong, S.; Reamtong, O.; Chongsa-Nguan, M.; Chaicumpa, W.; Indrawattana, N. Human single-chain variable fragments neutralize Pseudomonas aeruginosa quorum sensing molecule, 3O-C12-HSL, and prevent cells from the HSL-mediated apoptosis. Front. Microbiol. 2020, 11, 1172. [CrossRef]

41. Thayer, M.M.; Flaherty, K.M.; McKay, D.B. Three-dimensional structure of the elastase of Pseudomonas aeruginosa at 1.5- $\AA$ resolution. J. Biol. Chem. 1991, 266, 2864-2871. [CrossRef]

42. Roy, A.; Kucukural, A.; Zhang, Y. I-TASSER: A unified platform for automated protein structure and function prediction. Nature Prot. 2010, 5, 725-738. [CrossRef]

43. Yang, J.; Zhang, Y. I-TASSER server: New development for protein structure and function predictions. Nucleic Acids Res. 2015, 43, W174-W181. [CrossRef] [PubMed]

44. Xu, D.; Zhang, Y. Improving the physical realism and structural accuracy of protein models by a two-step atomic-level energy minimization. Biophys. J. 2011, 101, 2525-2534. [CrossRef]

45. Zhang, J.; Liang, Y.; Zhang, Y. Atomic-level protein structure refinement using fragment-guided molecular dynamics conformation sampling. Structure 2011, 19, 1784-1795. [CrossRef]

46. Brenke, R.; Hall, D.R.; Chuang, G.Y.; Comeau, S.R.; Bohnuud, T.; Beglov, D.; Schueler-Furman, O.; Vajda, S.; Kozakov, D. Application of asymmetric statistical potentials to antibody-protein docking. Bioinformatics 2012, 28, 2608-2614. [CrossRef]

47. Comeau, S.R.; Gatchell, D.W.; Vajda, S.; Camacho, C.J. ClusPro: A fully automated algorithm for protein-protein docking. Nucleic Acids Res. 2004, 32, W96-W99. [CrossRef] [PubMed]

48. Kozakov, D.; Hall, D.R.; Xia, B.; Porter, K.A.; Padhorny, D.; Yueh, C.; Beglov, D.; Vajda, S. The ClusPro web server for proteinprotein docking. Nature Protoc. 2017, 12, 255. [CrossRef] [PubMed]

49. Kessler, E.; Safrin, M. Synthesis, processing, and transport of Pseudomonas aeruginosa elastase. J. Bacteriol. 1988, $170,5241-5247$. [CrossRef]

50. Kessler, E.; Safrin, M.; Peretz, M.; Burstein, Y. Identification of cleavage sites involved in proteolytic processing of Pseudomonas aeruginosa preproelastase. FEBS Lett. 1992, 299, 291-293. [CrossRef]

51. McIver, K.S.; Kessler, E.; Ohman, D.E. Substitution of active-site His-223 in Pseudomonas aeruginosa elastase and expression of the mutated lasB alleles in Escherichia coli show evidence for autoproteolytic processing of proelastase. J. Bacteriol. 1991, 173, 7781-7789. [CrossRef] [PubMed]

52. McIver, K.S.; Kessler, E.; Olson, J.C.; Ohman, D.E. The elastase propeptide functions as an intramolecular chaperone required for elastase activity and secretion in Pseudomonas aeruginosa. Mol. Microbiol. 1995, 18, 877-889. [CrossRef]

53. Braun, P.; Tommassen, J.; Filloux, A. Role of the propeptide in folding and secretion of elastase of Pseudomonas aeruginosa. Mol. Microbiol. 1996, 19, 297-306. [CrossRef]

54. Cathcart, G.; Gilmore, B.; Walker, B. Enzyme Kinetic Measurements for a Combinatorial Library of Inhibitors of Pseudomonas Elastase. School of Pharmacy, QUB, Belfast. BMG LABTECH. Available online: https:/ / pure.qub.ac.uk. (accessed on 10 June 2021).

55. Padlan, E. A possible procedure for reducing the immunogenicity of antibody variable domains while preserving their ligandbinding properties. Mol. Immunol. 1991, 28, 489-498. [CrossRef] 
56. Shi, B.; Ma, L.; He, X.; Wang, X.; Wang, P.; Zhou, L.; Yao, X. Comparative analysis of human and mouse immunoglobulin variable heavy regions from IMGT/LIGM-DB with IMGT/HighV-QUEST. Theor. Biol. Med. Model. 2014, 11, 1-11. [CrossRef]

57. Rawlings, N.D.; Salvesen, G. Handbook of Proteolytic Enzymes, 3rd ed.; Academic Press: Amsterdam, The Netherlands, 2013; Volume 3.

58. Campa, M.; Bendinelli, M.; Friedman, H. Pseudomonas Aeruginosa As an Opportunistic Pathogen; Springer Science \& Business Media: New York, NY, USA, 2012. 